\title{
AMELIORATION OF SALINITY STRESS ON TRANSPLANT AMAN RICE THROUGH GREEN MANURE AND GYPSUM
}

\author{
M. Z. Siam, S. S. Hossain, A. K. Hassan and M. A. Kader \\ Department of Agronomy, Bangladesh Agricultural University, Mymensingh 2202, Bangladesh \\ Corresponding author: abdul.kader@bau.edu.bd
}

Key words: Salinity, aman rice, green manure, gypsum

\begin{abstract}
An experiment was conducted at the net house of Department of Agronomy, Bangladesh Agricultural University, Mymensingh from July to December 2012 to investigate the ameliorative effect of green manure and gypsum application on the yield of transplant Aman rice variety BRRI dhan 40 under various levels salinity stress. Sodium chloride induced salinity was imposed at tillering stage of plant development. The levels of salinity were 0,25 and $50 \mathrm{mM}$ $\mathrm{NaCl}$. Green manure @ 0,5 and $10 \mathrm{t} \mathrm{ha}^{-1}$ and gypsum @ 0 and $1 \mathrm{~g} \mathrm{~kg}^{-1}$ soil were applied to ameliorate the salinity stress effect. $1 \mathrm{~g} \mathrm{~kg}-1$ soil were applied to ameliorate the salinity stress effect. Results revealed that the different levels of salinity had significant adverse effect on plant height, number of tillers hill ${ }^{-1}$, number of effective tillers hill ${ }^{-1}$, number of ineffective tillers hill ${ }^{-1}$, panicle length, number of grains penicle ${ }^{-1}, 1000$-grain weight, grain yield, biological yield and harvest index (HI). All the plants were affected badly when they were exposed to salinity level of $50 \mathrm{mM} \mathrm{NaCl}$. Application of green manure and gypsum helped them ameiorate salinity either individually or in combination at all salinity levels. Grain yield reduction at $50 \mathrm{mM}$ salinity level was $38.64 \%$ compared to control which was minimized to $19.04 \%$ by the application of green manure @ $10 \mathrm{t} \mathrm{ha}^{-1}$. Grain yield reduction was also minimized from $37.08 \%$ to $27 \%$ at the same level of salinity by the application of gypsum@ $1 \mathrm{~g} \mathrm{~kg}^{-1}$ soil. Similar amelioration effect was also observed in case of straw yield. The amelioration was improved further when both green manure and gypsum were applied. Without any salinity stress grain yield was $4.49 \mathrm{t} \mathrm{ha}^{-1}$, which was reduced to $2.61 \mathrm{t} \mathrm{ha}^{-1}$ (41.87\% reduction) when the crop was stressed with $50 \mathrm{mM}$ salinity. Application of green manure@ $10 \mathrm{t} \mathrm{ha}^{-1}$ and gypsum @ $1 \mathrm{~g} \mathrm{~kg}^{-1}$ soil improved grain yield to 4.00 $\mathrm{t} \mathrm{ha} \mathrm{a}^{-1}$, where yield reduction was just $10.91 \%$. Similar improvement was also found in straw yield. The results of the study conclude that salinity stress in transplant Aman rice var. BRRI dhan40 could successfully be ameliorated through application of green manure@10 tha ${ }^{-1}$ and gypsum@1 $\mathrm{g} \mathrm{kg}^{-1}$ soil.
\end{abstract}

\section{Introduction}

One of the most common soil problems across the world is the salinity. However, out of 380 million hectare of saline soils on the earth surface, 240 million hectares are not strongly saline (Massoud, 1994) and this area can be converted to potential areas for crop production. Cultivation of these lands could improve the economic status of the small farmers in the belt of South and Southeast Asia. In Bangladesh, over $30 \%$ of the net cultivable area lies in the coastal area being affected by varying degrees of soil salinity (Karim et al., 1990). Agricultural land use in these areas is very poor and cropping intensity is very low. Salinity largely reduces the yield of rice in the coastal areas of Bangladesh. The salinity of these soils is either derived from tidal flooding with saline water at high spring tides or from sporadic inundation with salt water during cyclonic storm surges.

Rice is a salt sensitive crop species. Salinity level of $4 \mathrm{dsm}^{-1}$ is considered as critical level for rice. However, rice exhibits considerable intra-specific variability in tolerance to salinity (Flowers and Yeo, 1981). BRRI has been working for developing salt-tolerant rice variety for more than 30 years. For transplant Aman season, they have developed BRRI dhan40 and released in 2003. This variety can tolerate $8^{-} 10 \mathrm{dSm}^{-1}$ salinity when they are tender and $6 \mathrm{dSm}^{-1}$ salinity in rest of the living period. Salinity stress reduces the growth and yield of rice to a great extent (Hossain, 2002; Sen, 2002; Islam 
Siam et al.

et al., 2004; Rashid, 2005).

The permanent reclamation of the saline soils in Bangladesh is difficult and complex due to frequent inundation and tidal flooding. It would, therefore, be wise to grow the salt tolerant varieties of rice. Beside this, we need excellent inventories on salt-affected soils and development of cheaper options for amelioration of salinity. The amelioration of salinity stress basically involves replacing the sodium ions with more favorable calcium ions. Application of organic manure and gypsum could be an effective way to ameliorate the salinity stress in rice to a great extent. Use of green manures would pave a great path for reclamation of salinity. There is an intimate relationship between soil $\mathrm{pH}$, level of $\mathrm{CO}_{2}$ and calcium ion activity. It has been reported that the increasing amount of green manures in such soil facilitates rapid production of $\mathrm{CO}_{2}$ and enhances the soluble calcium status of soils (Vakeesan \& Nishanthan, 2007). That, in turn, replaces the sodium ions, resulting in the improvement of saline soil. Gypsum is another source of $\mathrm{Ca}^{2+}$. It replaces the $\mathrm{Na}^{+}$ion of soil. Ameliorative effect of gypsum application is reported in saline soil as well as in sodic soil (Khattak et al., 2007; Singh et al., 2008; Singh et al., 2009).

To meet the consequent increasing demand of the growing population in the country food production needs to be increased either by enhancing arable land or by increasing yield per hectare. But the scope of enhancing arable land is very limited in Bangladesh. So, food production must be increased by increasing yield per hectare to satisfy the consequent increased demand, particularly in the vulnerable areas of the country like salinity affected coastal area. Considering above facts, the present research was conducted to find out the ameliorative effect of green manure and gypsum at various levels of salinity stress on transplant Aman rice.

\section{Materials and Methods}

The research work was carried out at the nethouse of the Department of Agronomy, Bangladesh Agricultural University, Mymensingh from July to December, 2012 using green manure @ 0,5 and $10 \mathrm{t} \mathrm{ha}^{-1}$ and gypsum @ 0 and $1 \mathrm{~g} \mathrm{~kg}^{-1}$ soil to ameliorate salinity stress levels of 0,25 and $50 \mathrm{mM}$ $\mathrm{NaCl}$ in transplant Aman rice var. BRRI dhan40. The experiment was laid out in a Completely Randomized Design (CRD) with 3 (three) replications. The experimental soil was loamy in texture having a soil $\mathrm{pH}$ of 6.43, moderate in organic matter content. The soil was dried in sun and loosened and all the inert matter was removed. After that, the soil was filled in the Chari having $60 \mathrm{~cm}$ diameter on top and $40 \mathrm{~cm}$ in height. Each experimental Chari contained five hills of rice and denotes a replication. Seedlings were raised in well-prepared nursery bed. The applied fertilizer doses per Chari were urea 5.1 g, TSP $1.68 \mathrm{~g}$, MoP $2.24 \mathrm{~g}{ }^{1}$, gypsum $2.24 \mathrm{~g}$, and zinc sulphate $\left(\mathrm{ZnSO}_{4}\right) 0.035 \mathrm{~g}$. The total amount of TSP, MoP, gypsum and zinc sulphate $\left(\mathrm{ZnSO}_{4}\right)$ and one third of urea was applied during the final Chari preparation and the rest of urea was top dressed in two equal splits one at 20 days after transplanting (DAT) and one at 40 DAT. All the fertilizers were incorporated in the top most soil within 6 inch of the Chari soil. 30-day old seedlings were transplanted. Three seedlings were transplanted in each hill. Gap filling (after 1 week of transplanting), regular weeding (hand weeding), regular irrigation and all other intercultural operations were practiced timely. Salinity stress at the levels of 0,25 and $50 \mathrm{mM} \mathrm{NaCl}$ was imposed when the crop was at maximum tillering stage. Gypsum was applied as per treatment specification immediately after salinity stress imposition. The common agricultural practices for growing BRRI dhan 40 according to the recommendations of Bangladesh Rice Research Institute (BRRI) were followed. At maturity stage the crop was harvested treatment wise and data were collected on yield and yield parameters. The data were statistically analyzed according to Gomez and Gomez (1984). Least significant difference (LSD) was used for mean comparisons.

\section{Results and Discussion}

The results revealed that the green manure and gypsum exerted significant influence on the yield and yield contributing characters of Transplant Aman rice var. BRRI dhan40 under various levels of salinity stress. 
Salinity Stress on Transplant Aman Rice

\section{Effect of salinity stress}

The effect of salinity stress on yield and yield attributes of transplant Aman rice var. BRRI dhan40 was statistically significant (Table 1 ). It was observed that number of total tillers hill ${ }^{-1}$, effective tillers hill ${ }^{-1}$ and ineffective tillers hill $^{-1}$ decreased with the increasing salinity level. The highest no. of total tillers hill ${ }^{-1}$ (14.80), effective tillers hill $^{-1}$ (11.14), and ineffective tillers hill ${ }^{-1}$ (3.66) were observed at the control $\left(\mathrm{S}_{0}\right)$ salinity level. On the other hand, the lowest no. of total tillers hill ${ }^{-1}$ (13.09), no. of effective tillers hill $^{-1}$ (10.36) and ineffective tillers hill ${ }^{-1}$ (2.76) were recorded at the 50 $\mathrm{mM} \mathrm{NaCl}\left(\mathrm{S}_{2}\right)$ salinity level. Besides, panicle length, no. of grains panicle ${ }^{-1}$, grain yield and straw yield varied significant due to salinity stress. Panicle length, no. of total spikelets panicle ${ }^{-1}$, no. of grains panicle $^{-1}$, grain yield and straw yield decreased with the increasing salinity levels. The highest panicle length $(24.31 \mathrm{~cm})$, no. of filled grains panicle ${ }^{-1}(118.1), 1000$-grain weight $(24.45 \mathrm{~g})$, grain yield (4.88 $\left.\mathrm{t} \mathrm{ha}^{-1}\right)$ and straw yield $\left(6.37 \mathrm{t} \mathrm{ha}^{-1}\right)$ were observed at the control $\left(\mathrm{S}_{0}\right)$ salinity level and the lowest panicle length (23.51), no. of grains panicle ${ }^{-1}$ (74.00), 1000-grain weight (24.18 g), grain yield $\left(3.22 \mathrm{t} \mathrm{ha}^{-1}\right)$ and straw yield $\left(4.14 \mathrm{t} \mathrm{ha}^{-1}\right)$ were observed at the $50 \mathrm{mM} \mathrm{NaCl}\left(\mathrm{S}_{2}\right)$ salinity level. The no. of sterile spikelets panicle ${ }^{-1}$ increased with the increasing salinity levels. The highest no. of sterile spikelets panicle ${ }^{-1}$ (28.81) was observed at the $50 \mathrm{mM} \mathrm{NaCl}\left(\mathrm{S}_{2}\right)$ salinity level and the lowest no. of sterile spikelets panicle ${ }^{-1}(15.95)$ was observed at control $\left(\mathrm{S}_{0}\right)$ salinity level. So, the salinity stress@25mM \& 50 mM NaCl had significant negative impact on transplant Aman rice. The similar result was previously reported by Hossain (2002), Sen (2002), Islam et al. (2004) and Rashid (2005).

Table 1. Effect of salinity stress on yield and yield attributes of transplant Aman rice var. BRRI dhan40

\begin{tabular}{|c|c|c|c|c|c|c|c|c|c|}
\hline $\begin{array}{l}\text { Salinity } \\
\text { stress }\end{array}$ & $\begin{array}{c}\text { No. of } \\
\text { total tillers } \\
\text { hill }^{-1}\end{array}$ & $\begin{array}{c}\text { No. of } \\
\text { effective } \\
\text { tillers } \\
\text { hill }^{-1}\end{array}$ & $\begin{array}{c}\text { No. of non } \\
\text { effective } \\
\text { tillers } \\
\text { hill }^{-1}\end{array}$ & $\begin{array}{l}\text { Panicle } \\
\text { length } \\
(\mathrm{cm})\end{array}$ & $\begin{array}{c}\text { No. of } \\
\text { grains } \\
\text { panicle }^{-1}\end{array}$ & $\begin{array}{c}\text { No. of } \\
\text { sterile } \\
\text { spikelets } \\
\text { panicle }^{-1}\end{array}$ & $\begin{array}{c}1000- \\
\text { grain } \\
\text { weight } \\
\text { (g) }\end{array}$ & $\begin{array}{c}\text { Grain } \\
\text { yield } \\
\left(\mathrm{t} \mathrm{ha}^{-1}\right)\end{array}$ & $\begin{array}{c}\text { Straw } \\
\text { yield } \\
\left(\mathrm{t} \mathrm{ha}^{-1}\right)\end{array}$ \\
\hline $\mathrm{S}_{0}$ & $14.80 \mathrm{a}$ & $11.14 \mathrm{a}$ & $3.66 \mathrm{a}$ & $24.31 \mathrm{a}$ & $102.2 \mathrm{a}$ & $15.95 \mathrm{c}$ & $24.45 \mathrm{a}$ & $4.88 \mathrm{a}$ & $6.37 \mathrm{a}$ \\
\hline $\mathrm{S}_{1}$ & $14.04 \mathrm{~b}$ & $10.81 \mathrm{~b}$ & $3.18 \mathrm{~b}$ & $23.96 \mathrm{~b}$ & 91.27 b & $22.16 \mathrm{~b}$ & 24.32ab & $4.25 \mathrm{~b}$ & $5.64 \mathrm{~b}$ \\
\hline $\mathrm{S}_{2}$ & 13.09 c & 10.36 c & $2.76 \mathrm{c}$ & $23.51 \mathrm{c}$ & 74.00 c & $28.81 \mathrm{a}$ & $24.18 \mathrm{~b}$ & $3.22 \mathrm{c}$ & $4.14 \mathrm{c}$ \\
\hline F-test & $* *$ & ** & ** & ** & ** & $* *$ & * & ** & ** \\
\hline $\operatorname{LSD}_{(0 .}$ & 0.052 & 0.042 & 0.047 & 0.204 & 0.324 & 0.317 & 0.196 & 0.030 & 0.047 \\
\hline CV (\%) & 0.55 & 0.57 & 2.20 & 1.26 & 0.54 & 2.10 & 1.19 & 1.11 & 1.25 \\
\hline
\end{tabular}

In a column, figures with same letter or without letter do not differ significantly, whereas figures with dissimilar letters differ significantly (as per DMRT)

* $=$ Significant at $5 \%$ level of probability

$* *$ = Significant at $1 \%$ level of probability

NS $=$ Not significant

$\mathrm{S}_{0}=0 \mathrm{mM} \mathrm{NaCl}, \mathrm{S}_{1}=25 \mathrm{mM} \mathrm{NaCl}, \mathrm{S}_{2}=50 \mathrm{mM} \mathrm{NaCl}$

\section{Amelioration of salinity stress through green manuring}

Green manuring exerted significant influence on yield and yield attributes of transplant Aman rice var. BRRI dhan40 at different levels of salinity stress (Table 2). The interaction effect of salinity levels and green manure application on number of total tillers hill ${ }^{-1}$, number of effective tillers hill $^{-1}$, number of ineffective tillers hill $^{-1}$, number of total grain panicle ${ }^{-1}$, number of filled grain panicle ${ }^{-1}$, grain yield and straw yield were found statistically significant. The number of total tillers hill ${ }^{-1}$, effective tillers hill ${ }^{-1}$, ineffective tillers hill $^{-1}$, number of total grain panicle ${ }^{-1}$, number of filled grain panicle ${ }^{-1}$, grain weight, straw yield, biological yield decreased with increasing salinity level and increased with increasing green manure level. The highest number of tillers (15.19), effective tillers hill ${ }^{-1}$ (11.35), number of ineffective tillers hill ${ }^{-1}$ (3.83), number of grain panicle ${ }^{-1}$ (121.2), number of grain panicle ${ }^{-1}$ (108.1), grain yield (5.24 $\mathrm{t} \mathrm{ha}^{-1}$ ) and straw yield (6.70) were recorded at control salinity(S0)x $10 \mathrm{t} \mathrm{ha}^{-}$ 
Siam et al.

${ }^{1}$ green manure. The lowest number of tillers (12.80), number of effective tillers hill ${ }^{-1}(10.15)$, ineffective tillers hill $^{-1}(2.65)$, number of grain panicle ${ }^{-1}$ (98.65), number of grain panicle ${ }^{-1}$ (66.78), grain yield (2.83 tha $\mathrm{th}^{-1}$ ) and straw yield (3.62) were observed in $50 \mathrm{mM}$ salinity level x no green manure application. Table 3 shows reduction of grain and straw yields of transplant Aman rice var. BRRI dhan 40 under various levels of salinity stress over control and under application of green manure.

Table 2. Interaction effect of green manure and salinity stress on yield and yield attributes of transplant Aman rice var. BRRI dhan 40

\begin{tabular}{|c|c|c|c|c|c|c|c|c|c|}
\hline $\begin{array}{l}\text { Interaction } \\
(\mathrm{M} \times \mathrm{S})\end{array}$ & $\begin{array}{c}\text { Total tillers } \\
\text { hill }^{-1}\end{array}$ & $\begin{array}{c}\text { No. of } \\
\text { effective } \\
\text { tillers } \\
\text { hill }^{-1}\end{array}$ & \begin{tabular}{|c|}
$\begin{array}{c}\text { No. of non } \\
\text { effective } \\
\text { tillers } \\
{ }_{1} \text { hill- }\end{array}$ \\
\end{tabular} & $\begin{array}{l}\text { Panicle } \\
\text { length } \\
\text { (cm) }\end{array}$ & $\begin{array}{c}\text { No. of } \\
\text { grains } \\
\text { panicle }^{-1}\end{array}$ & \begin{tabular}{|c|} 
No. of \\
sterile \\
spikelets $^{-1}$ \\
panicle $^{-1}$ \\
\end{tabular} & $\begin{array}{c}1000- \\
\text { grain } \\
\text { weight } \\
\text { (g) }\end{array}$ & $\begin{array}{c}\text { Grain } \\
\text { yield } \\
\left(\mathrm{t} \mathrm{ha}^{-1}\right)\end{array}$ & $\begin{array}{c}\text { Straw } \\
\text { yield } \\
\left(\mathrm{t} \mathrm{ha}^{-1}\right)\end{array}$ \\
\hline $\mathrm{M}_{0} \mathrm{~S}_{0}$ & $14.43 \mathrm{c}$ & $10.96 \mathrm{c}$ & $3.46 \mathrm{c}$ & 24.11 & $98.21 \mathrm{c}$ & $17.61 \mathrm{~g}$ & 24.38 & $4.62 \mathrm{c}$ & $6.10 \mathrm{c}$ \\
\hline $\mathrm{M}_{0} \mathrm{~S}_{1}$ & $13.84 \mathrm{f}$ & $10.71 \mathrm{~d}$ & $3.11 \mathrm{e}$ & 23.84 & $87.07 \mathrm{f}$ & $24.18 \mathrm{~d}$ & 24.22 & 3.98 e & $5.21 \mathrm{e}$ \\
\hline $\mathrm{M}_{0} \mathrm{~S}_{2}$ & $12.80 \mathrm{i}$ & $10.15 \mathrm{f}$ & $2.65 \mathrm{~h}$ & 23.35 & $66.78 \mathrm{i}$ & 31.88 a & 24.24 & $2.83 \mathrm{~h}$ & $3.62 \mathrm{~h}$ \\
\hline $\mathrm{M}_{1} \mathrm{~S}_{0}$ & $14.80 \mathrm{~b}$ & $11.10 \mathrm{~b}$ & $3.70 \mathrm{~b}$ & 24.40 & $100.2 \mathrm{~b}$ & $17.17 \mathrm{~g}$ & 24.42 & $4.80 \mathrm{~b}$ & $6.30 \mathrm{~b}$ \\
\hline $\mathrm{M}_{1} \mathrm{~S}_{1}$ & $14.03 \mathrm{e}$ & $10.75 \mathrm{~d}$ & $3.17 \mathrm{e}$ & 23.86 & 89.90 e & $22.51 \mathrm{e}$ & 24.31 & $4.19 \mathrm{~d}$ & $5.50 \mathrm{~d}$ \\
\hline $\mathrm{M}_{1} \mathrm{~S}_{2}$ & $12.99 \mathrm{~h}$ & $10.23 \mathrm{e}$ & $2.76 \mathrm{~g}$ & 23.55 & $72.57 \mathrm{~h}$ & $29.51 \mathrm{~b}$ & 23.89 & $3.10 \mathrm{~g}$ & $4.00 \mathrm{~g}$ \\
\hline $\mathrm{M}_{2} \mathrm{~S}_{0}$ & $15.19 \mathrm{a}$ & 11.35 a & $3.83 \mathrm{a}$ & 24.42 & $108.1 \mathrm{a}$ & $13.06 \mathrm{~h}$ & 24.55 & $5.24 \mathrm{a}$ & $6.70 \mathrm{a}$ \\
\hline $\mathrm{M}_{2} \mathrm{~S}_{1}$ & $14.24 \mathrm{~d}$ & 10.98 c & $3.27 \mathrm{~d}$ & 24.17 & $96.83 \mathrm{~d}$ & $19.78 \mathrm{f}$ & 24.42 & $4.58 \mathrm{c}$ & $6.23 \mathrm{~b}$ \\
\hline $\mathrm{M}_{2} \mathrm{~S}_{2}$ & $13.48 \mathrm{~g}$ & $10.70 \mathrm{~d}$ & $2.88 \mathrm{f}$ & 23.65 & $82.67 \mathrm{~g}$ & 25.02 c & 24.42 & $3.74 \mathrm{f}$ & $4.81 \mathrm{f}$ \\
\hline F-test & $* *$ & $* *$ & $* *$ & NS & $* *$ & $* *$ & NS & $* *$ & $* *$ \\
\hline $\operatorname{LSD}_{(0.05)}$ & 0.090 & 0.074 & 0.082 & - & 0.561 & 0.550 & - & 0.052 & 0.083 \\
\hline CV (\%) & 0.55 & 0.57 & 2.20 & 1.26 & 0.54 & 2.10 & 1.19 & 1.11 & 1.25 \\
\hline
\end{tabular}

In a column, figures with same letter or without letter do not differ significantly, whereas figures with dissimilar letters differ significantly (as per DMRT)

* = Significant at $5 \%$ level of probability

** $=$ Significant at $1 \%$ level of probability

NS $=$ Not significant

$\mathrm{M}_{0}=0, \mathrm{M}_{1}=5$ ton green manure $/ \mathrm{ha}, \mathrm{M}_{2}=10$ ton green manure $/ \mathrm{ha}, \mathrm{S}_{0}=0 \mathrm{mM} \mathrm{NaCl}, \mathrm{S}_{1}=25 \mathrm{mM} \mathrm{NaCl}, \mathrm{S}_{2}=50 \mathrm{mM} \mathrm{NaCl}$

It is evident from the table that application of green manure @ $5 \mathrm{t} \mathrm{ha}^{-1}$ and $10 \mathrm{t} \mathrm{ha}^{-1}$ increased grain yield by $3.89 \%$ and $13.53 \%$ over control when no salinity was imposed. On the other hand, imposition of salinity stress @25 mM NaCl and $50 \mathrm{mM} \mathrm{NaCl}$ decreased grain yield by $13.85 \%$ and 38.64\%, respectively. Over control, the grain yield reduction of $13.85 \%$ was minimized to less than $1 \%$ and that of $38.64 \%$ to $19.05 \%$ by applying green manure @ $10 \mathrm{t} \mathrm{ha}^{-1}$. In case of straw yield, application of green manure@ $5 \mathrm{t} \mathrm{ha}^{-1}$ and $10 \mathrm{t} \mathrm{ha}^{-1}$ increased straw yield by 3.27\% and 9.74\%, respectively over control when no salinity was imposed. On the other hand, imposition of salinity stress @25 mM NaCl and 50 mM $\mathrm{NaCl}$ decreased straw yield by $14.57 \%$ and $40.57 \%$, respectively. Over control the straw yield reduction of $14.57 \%$ was minimized to yield increase of $2.04 \%$ and that of $40.57 \%$ to $21.21 \%$ by applying green manure @ $10 \mathrm{t} \mathrm{ha}^{-1}$. The results revealed that the ameliorative effect of green manure at higher dose $(10 \mathrm{t}$ $\mathrm{ha}^{-1}$ ) was more effective than the lower dose $\left(5 \mathrm{t} \mathrm{ha}^{-1}\right)$ at the both salinity levels used in the study.

Table 3. Amelioration of salinity stress on grain yield and straw yields of transplant Aman rice var. BRRI dhan 40 by green manuring

\begin{tabular}{c|c|c|c|c}
\hline Interaction (M x S) & $\begin{array}{c}\text { Grain yield } \\
\left(\mathrm{t} \mathrm{ha}^{-1}\right)\end{array}$ & $\begin{array}{c}\text { \%increase or } \\
\text { decrease over } \\
\text { control }\end{array}$ & $\begin{array}{c}\text { Straw yield } \\
\left(\mathrm{t} \mathrm{ha}^{-1}\right)\end{array}$ & $\begin{array}{c}\text { \%increase or } \\
\text { decrease over } \\
\text { control }\end{array}$ \\
\hline $\mathrm{M}_{0} \mathrm{~S}_{0}$ & 4.62 & - & 6.105 & - \\
$\mathrm{M}_{1} \mathrm{~S}_{0}$ & 4.8 & 3.89 & 6.305 & 3.27
\end{tabular}


Salinity Stress on Transplant Aman Rice

\begin{tabular}{lcccc}
$\mathrm{M}_{2} \mathrm{~S}_{0}$ & 5.24 & 13.52 & 6.7 & 9.74 \\
$\mathrm{M}_{0} \mathrm{~S}_{1}$ & 3.98 & -13.85 & 5.215 & -14.57 \\
$\mathrm{M}_{1} \mathrm{~S}_{1}$ & 4.19 & -9.30 & 5.5 & -9.90 \\
$\mathrm{M}_{2} \mathrm{~S}_{1}$ & 4.58 & -0.75 & 6.23 & 2.04 \\
$\mathrm{M}_{0} \mathrm{~S}_{2}$ & 2.83 & -38.63 & 3.628 & -40.57 \\
$\mathrm{M}_{1} \mathrm{~S}_{2}$ & 3.10 & -32.79 & 4.005 & -34.39 \\
$\mathrm{M}_{2} \mathrm{~S}_{2}$ & 3.74 & -19.04 & 4.81 & -21.21 \\
\hline
\end{tabular}

$\mathrm{M}_{0}=0, \mathrm{M}_{1}=5$ ton green manure $/ \mathrm{ha}, \mathrm{M}_{2}=10$ ton green manure $/ \mathrm{ha}$

$\mathrm{S}_{0}=0 \mathrm{mM} \mathrm{NaCl} . \mathrm{S}_{1}=25 \mathrm{mM} \mathrm{NaCl}, \mathrm{S}_{2}=50 \mathrm{mM} \mathrm{NaCl}$

\section{Amelioration of salinity stress through gypsum application}

As evident in Table 4, the effect of gypsum application was significant on yield and yield components of transplant Aman rice var. BRRI dhan40 under different levels of salinity stress. The highest number of total tillers hill ${ }^{-1}$ (14.97), number of effective tillers hill $^{-1}(11.21)$, number of ineffective tillers hill ${ }^{-1}$ (3.76), panicle length $(24.42 \mathrm{~cm})$, number of total spikelets-1 (119.1), number of grains panicle $^{-1}$ (104.5), 1000-grain weight (24.49 g), grain yield (5.02 t ha-1) and straw yield (6.52 $\left.\mathrm{t} \mathrm{ha}^{-1}\right)$ were recorded in no salinity stress condition and $1 \mathrm{~g}_{\text {gypsum }} \mathrm{kg}^{-1}$ soil application. On the other hand, the lowest number of total tillers (12.85), effective tillers hill ${ }^{-1}(10.23)$, ineffective tillers hill $^{-1}$ (2.68), panicle length $(23.44 \mathrm{~cm})$, number of total spikelets panicle ${ }^{-1}(100.7)$, number of grains panicle $^{-1}$ (70.28), 1000- grain weight (24.04 g), grain yield (2.99 $\left.\mathrm{t} \mathrm{ha}^{-1}\right)$, straw yield (3.83 $\left.\mathrm{t} \mathrm{ha}^{-1}\right)$ were obtained from $50 \mathrm{mM}$ salinity level x no gypsum application. Table 5 indicates reduction in grain and straw yields of transplant Aman rice var. BRRI dhan 40 under various levels of salinity stress over control and under application of gypsum. It is evident from the table 5 that application of gypsum @ $1 \mathrm{~g} \mathrm{~kg}^{-1}$ soil increased grain yield by $5.52 \%$ over control when no salinity was imposed. On the other hand, salinity stress@25 mM NaCl and 50 mM NaCl decreased grain yield by 13.66\% and 37.08\%, respectively. The grain yield reduction of $13.66 \%$ was curtailed to less than $7.56 \%$ and that of $37.08 \%$ to $27.26 \%$ over control by applying gypsum @ $1 \mathrm{~g} \mathrm{~kg}^{-1}$ soil. In case of straw yield, application of gypsum @ $1 \mathrm{~g} \mathrm{~kg}^{-1}$ soil augmented straw yield by $5.05 \%$ over control when there is no salinity. Again, imposition of salinity stress @25 mM NaCl and 50 mM NaCl curtailed straw yield by 13.24\% and 38.35\%, respectively. Over control the straw yield reduction of $13.24 \%$ was reduced to yield increase of $4.92 \%$ and that of $38.35 \%$ to $28.16 \%$ by applying gypsum @ $1 \mathrm{~g} \mathrm{~kg}^{-1}$ soil. The results revealed that the ameliorative effect of gypsum at higher dose $\left(1 \mathrm{~g} \mathrm{~kg}^{-1}\right.$ soil) was more effective than the control at the both salinity levels used in the study.

Table 4. Interaction effect of gypsum and salinity stress on yield and yield attributes of transplant Aman rice var. BRRI dhan 40

\begin{tabular}{l|c|c|c|c|c|c|c|c|c}
\hline $\begin{array}{l}\text { Interaction } \\
(\mathrm{G} \mathrm{x} \mathrm{S})\end{array}$ & $\begin{array}{c}\text { Total } \\
\text { tillers } \\
\text { hill }^{-1}\end{array}$ & $\begin{array}{c}\text { No. of } \\
\text { effective } \\
\text { tillers } \\
\text { hill }^{-1}\end{array}$ & $\begin{array}{c}\text { No. of non } \\
\text { effective } \\
\text { tillers } \\
\text { hill }^{-1}\end{array}$ & $\begin{array}{c}\text { Panicle } \\
\text { length } \\
(\mathrm{cm})\end{array}$ & $\begin{array}{c}\text { No. of } \\
\text { grains } \\
\text { panicle }^{-1}\end{array}$ & $\begin{array}{c}\text { No. of } \\
\text { sterile } \\
\text { spikelets } \\
\text { panicle }^{-1}\end{array}$ & $\begin{array}{c}1000- \\
\text { grain } \\
\text { weight } \\
(\mathrm{g})\end{array}$ & $\begin{array}{c}\text { Grain } \\
\text { yield } \\
\left(\mathrm{t} \mathrm{ha}^{-1}\right)\end{array}$ & $\begin{array}{c}\text { Straw } \\
\text { yield } \\
\left(\mathrm{t} \mathrm{ha}^{-1}\right)\end{array}$ \\
\hline $\mathrm{G}_{0} \mathrm{~S}_{0}$ & $14.64 \mathrm{~b}$ & $11.07 \mathrm{~b}$ & $3.57 \mathrm{~b}$ & 24.20 & $99.87 \mathrm{~b}$ & $17.28 \mathrm{e}$ & 24.41 & $4.75 \mathrm{~b}$ & $6.21 \mathrm{~b}$ \\
$\mathrm{G}_{0} \mathrm{~S}_{1}$ & $13.93 \mathrm{~d}$ & $10.72 \mathrm{~d}$ & $3.15 \mathrm{c}$ & 23.94 & $89.17 \mathrm{~d}$ & $23.20 \mathrm{c}$ & 24.27 & $4.107 \mathrm{~d}$ & $5.39 \mathrm{~d}$ \\
$\mathrm{G}_{0} \mathrm{~S}_{2}$ & $12.85 \mathrm{f}$ & $10.23 \mathrm{f}$ & $2.68 \mathrm{e}$ & 23.44 & $70.28 \mathrm{f}$ & $30.37 \mathrm{a}$ & 24.04 & $2.99 \mathrm{f}$ & $3.83 \mathrm{f}$ \\
$\mathrm{G}_{1} \mathrm{~S}_{0}$ & $14.97 \mathrm{a}$ & $11.21 \mathrm{a}$ & $3.76 \mathrm{a}$ & 24.42 & $104.5 \mathrm{a}$ & $14.61 \mathrm{f}$ & 24.49 & $5.02 \mathrm{a}$ & $6.52 \mathrm{a}$ \\
$\mathrm{G}_{1} \mathrm{~S}_{1}$ & $14.15 \mathrm{c}$ & $10.90 \mathrm{c}$ & $3.21 \mathrm{c}$ & 23.98 & $93.36 \mathrm{c}$ & $21.12 \mathrm{~d}$ & 24.37 & $4.39 \mathrm{c}$ & $5.90 \mathrm{c}$ \\
$\mathrm{G}_{1} \mathrm{~S}_{2}$ & $13.33 \mathrm{e}$ & $10.49 \mathrm{e}$ & $2.84 \mathrm{~d}$ & 23.58 & $77.73 \mathrm{e}$ & $27.24 \mathrm{~b}$ & 24.32 & $3.46 \mathrm{e}$ & $4.46 \mathrm{e}$ \\
$\mathrm{F}_{-}$test & $* *$ & $* *$ & $*$ & NS & $* *$ & $* *$ & NS & $* *$ & $* *$ \\
\hline $\mathrm{LSD}_{(0.05)}$ & 0.074 & 0.060 & 0.067 & - & 0.458 & 0.449 & - & 0.042 & 0.067 \\
$\mathrm{CV}(\%)$ & 0.55 & 0.57 & 2.20 & 1.26 & 0.54 & 2.10 & 1.19 & 1.11 & 1.25 \\
\hline
\end{tabular}


Siam et al.

In a column, figures with same letter or without letter do not differ significantly, whereas figures with dissimilar letters differ significantly (as per DMRT)

* = Significant at $5 \%$ level of probability

** $=$ Significant at $1 \%$ level of probability

NS = Not significant

$\mathrm{G}_{0}=0 \mathrm{~g}$ gypsum $/ \mathrm{kg}$ soil, $\mathrm{G}_{1}=1 \mathrm{~g}$ gypsum $/ \mathrm{kg}$ soil

$\mathrm{S}_{0}=0 \mathrm{mM} \mathrm{NaCl}, \mathrm{S}_{1}=25 \mathrm{mM} \mathrm{NaCl}, \mathrm{S}_{2}=50 \mathrm{mM} \mathrm{NaCl}$

Table 5. Amelioration of salinity stress on grain yield and straw yields of transplant Aman rice var. BRRI dhan 40 by gypsum application

\begin{tabular}{c|c|c|c|c}
\hline $\begin{array}{c}\text { Interaction } \\
(\mathrm{G} \mathrm{x} \mathrm{S})\end{array}$ & $\begin{array}{c}\text { Grain yield } \\
\left(\mathrm{t} \mathrm{ha}^{-1}\right)\end{array}$ & $\begin{array}{c}\text { \%increase or decrease over } \\
\text { control }\end{array}$ & $\begin{array}{c}\text { Straw yield } \\
\left(\mathrm{t} \mathrm{ha}^{-1}\right)\end{array}$ & $\begin{array}{c}\text { \%increase or decrease over } \\
\text { control }\end{array}$ \\
\hline $\mathrm{G}_{0} \mathrm{~S}_{0}$ & 4.75 & - & 6.21 & - \\
$\mathrm{G}_{1} \mathrm{~S}_{0}$ & 5.02 & 5.52 & 6.52 & 5.05 \\
$\mathrm{G}_{0} \mathrm{~S}_{1}$ & 4.10 & -13.66 & 5.39 & -13.24 \\
$\mathrm{G}_{1} \mathrm{~S}_{1}$ & 4.39 & -7.56 & 5.90 & -4.92 \\
$\mathrm{G}_{0} \mathrm{~S}_{2}$ & 2.99 & -37.08 & 3.83 & -38.35 \\
$\mathrm{G}_{1} \mathrm{~S}_{2}$ & 3.46 & -27.26 & 4.46 & -28.16 \\
\hline
\end{tabular}

$\mathrm{S}_{0}=0 \mathrm{mM} \mathrm{NaCl}, \mathrm{S}_{1}=25 \mathrm{mM} \mathrm{NaCl}, \mathrm{S}_{2}=50 \mathrm{mM} \mathrm{NaCl}$

$\mathrm{G}_{0}=0 \mathrm{~g}$ gypsum $/ \mathrm{kg}$ soil, $\mathrm{G}_{1}=1 \mathrm{~g}$ gypsum $/ \mathrm{kg}$ soil

\section{Amelioration of salinity stress through green manuring and gypsum application}

Interaction effect of gypsum and green manure was statistically significant on the yield and yield components of transplant Aman rice under various levels of salinity stress (Table 6). The highest number of tillers hill ${ }^{-1}$ (15.25), number of effective tillers hill ${ }^{-1}$ (11.40), number of ineffective tillers hill $^{-1}$ (3.85), panicle length $(24.53 \mathrm{~cm})$, number of grain panicle ${ }^{-1}(122.5)$, grain yield $\left(5.35 \mathrm{t} \mathrm{ha}^{-1}\right)$ and straw yield $\left(6.80 \mathrm{t} \mathrm{ha}^{-1}\right)$ were recorded when there was no salinity stress in the crops with application of $1 \mathrm{~g}$ gypsum $\mathrm{kg}^{-1}$ soil plus $10 \mathrm{t} \mathrm{ha}^{-1}$ green manure. In contrast, the lowest number of tillers (12.50), number of effective tillers hill $^{-1}(10.00)$, number of ineffective tillers hill $^{-1}(2.50)$, panicle length $(23.30 \mathrm{~cm})$, number of grain panicle ${ }^{-1}(63.41)$, grain yield $\left(2.61 \mathrm{t} \mathrm{ha}^{-1}\right)$ and straw yield $(3.30 \mathrm{t}$ $\mathrm{ha}^{-1}$ ) were found at $50 \mathrm{mM}$ salinity level with no gypsum application and no green manure application. Table 7 showed the reduction in grain and straw yields of transplant Aman rice var. BRRI dhan40 under various levels of salinity stress over control and under application of green manure. It perceived from the

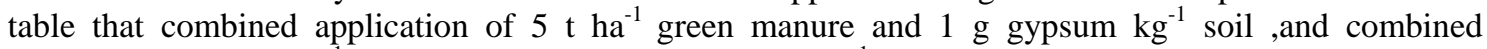
application of $10 \mathrm{t} \mathrm{ha}^{-1}$ green manure and $1 \mathrm{~g}$ gypsum kg-1 soil increased grain yield by $10.46 \%$ and $19.15 \%$ over control when no salinity was imposed. On the other hand, salinity stress @25 mM NaCl and $50 \mathrm{mM} \mathrm{NaCl}$ decreased grain yield by $14.03 \%$ and $41.87 \%$, respectively. Over control, the grain yield reduction of $14.03 \%$ was turned into an increase yield of $4 \%$ and that of $41.87 \%$ to $10.91 \%$ by combined application of green manure @ $10 \mathrm{t} \mathrm{ha}^{-1}$ and gypsum @ $1 \mathrm{~g} \mathrm{~kg}^{-1}$ soil. In case of straw yield, combined application of $5 \mathrm{t} \mathrm{ha}^{-1}$ green manure and $1 \mathrm{~g}_{\text {gypsum } \mathrm{kg}^{-1}}$ soil and combined application of $10 \mathrm{t} \mathrm{ha}^{-1}$ green manure and $1 \mathrm{~g}_{\text {gypsum kg}}{ }^{-1}$ soil increased straw yield by $9.62 \%$ and $14.86 \%$ over control when there is no salinity imposed. Again, imposition of salinity stress@25 mM NaCl and $50 \mathrm{mM} \mathrm{NaCl}$ decreased straw yield by $14.52 \%$ and $44.13 \%$, respectively. The straw yield reduction of $14.52 \%$ was turned into an increase yield of $7.60 \%$ and that of $44.13 \%$ to $15.37 \%$ by combined application of green manure @ $10 \mathrm{t}$ $\mathrm{ha}^{-1}$ and gypsum @1 $\mathrm{g} \mathrm{kg}^{-1}$ soil. The results revealed that the ameliorative effect of combined application of $10 \mathrm{t} \mathrm{ha}^{-1}$ green manure and $1 \mathrm{~g}_{\text {gypsum }} \mathrm{kg}^{-1}$ soil was more effective than the combined application of 5 $\mathrm{t} \mathrm{ha}{ }^{-1}$ green manure and $1 \mathrm{~g}_{\text {gypsum } \mathrm{kg}^{-1}}$ soil at the both salinity levels used in the study.

Table 6. Interaction effect of green manure, gypsum and salinity stress on yield and yield attributes of transplant Aman rice var. BRRI dhan40 
Salinity Stress on Transplant Aman Rice

\begin{tabular}{|c|c|c|c|c|c|c|c|c|c|}
\hline $\begin{array}{l}\text { Interaction } \\
(\mathrm{M} \times \mathrm{G} \times \mathrm{S})\end{array}$ & $\begin{array}{l}\text { Total } \\
\text { tillers } \\
\text { hill }^{-1}\end{array}$ & $\begin{array}{c}\text { No. of } \\
\text { effective } \\
\text { tillers } \\
\text { hill }^{-1}\end{array}$ & $\begin{array}{l}\text { No. of non } \\
\text { effective } \\
\text { tillers } \\
\text { hill }^{-1}\end{array}$ & $\begin{array}{c}\text { Panicle } \\
\text { length } \\
(\mathrm{cm})\end{array}$ & $\begin{array}{c}\text { No. of } \\
\text { grains } \\
\text { panicle }^{-1}\end{array}$ & $\begin{array}{c}\text { No. of } \\
\text { sterile } \\
\text { spikelets } \\
\text { panicle }^{-1}\end{array}$ & $\begin{array}{l}\text { 1000-grain } \\
\text { weight } \\
\text { (g) }\end{array}$ & $\begin{array}{l}\text { Grain } \\
\text { yield } \\
\left(\mathrm{t} \mathrm{ha}^{-1}\right)\end{array}$ & $\begin{array}{c}\text { Straw } \\
\text { yield } \\
\left(\mathrm{t} \mathrm{ha}^{-1}\right)\end{array}$ \\
\hline $\mathrm{M}_{0} \mathrm{G}_{0} \mathrm{~S}_{0}$ & 14.20 & 10.90 & $3.30 \mathrm{c}$ & 24.00 & $96.60 \mathrm{f}$ & $19.21 \mathrm{i}$ & $24.32 \mathrm{abc}$ & $4.49 \mathrm{fg}$ & $\overline{5.92 \mathrm{f}}$ \\
\hline $\mathrm{M}_{0} \mathrm{G}_{0} \mathrm{~S}_{1}$ & 13.70 & 10.60 & $3.10 \mathrm{~d}$ & 23.80 & $85.52 \mathrm{k}$ & $25.13 \mathrm{f}$ & $24.20 \mathrm{bcd}$ & $3.86 \mathrm{j}$ & $5.06 \mathrm{~h}$ \\
\hline $\mathrm{M}_{0} \mathrm{G}_{0} \mathrm{~S}_{2}$ & 12.50 & 10.00 & $2.50 \mathrm{~g}$ & 23.30 & $63.41 \mathrm{p}$ & 33.08 a & $23.70 \mathrm{~d}$ & 2.610 & $3.30 \mathrm{~m}$ \\
\hline $\mathrm{M}_{0} \mathrm{G}_{1} \mathrm{~S}_{0}$ & 14.65 & 11.02 & $3.63 \mathrm{~b}$ & 24.22 & $99.82 \mathrm{~d}$ & $16.01 \mathrm{j}$ & $24.43 \mathrm{abc}$ & $4.75 \mathrm{~d}$ & $6.29 \mathrm{c}$ \\
\hline $\mathrm{M}_{0} \mathrm{G}_{1} \mathrm{~S}_{1}$ & 13.97 & 10.80 & $3.12 \mathrm{~d}$ & 23.89 & 88.61 i & $23.24 \mathrm{~g}$ & 24.24abcd & $4.10 \mathrm{~h}$ & $5.37 \mathrm{~g}$ \\
\hline $\mathrm{M}_{0} \mathrm{G}_{1} \mathrm{~S}_{2}$ & 13.10 & 10.30 & $2.80 \mathrm{ef}$ & 23.40 & $70.14 \mathrm{n}$ & $30.68 c$ & 24.78 a & $3.06 \mathrm{~m}$ & $3.95 \mathrm{k}$ \\
\hline $\mathrm{M}_{1} \mathrm{G}_{0} \mathrm{~S}_{0}$ & 14.60 & 11.00 & $3.60 \mathrm{~b}$ & 24.29 & $97.31 \mathrm{f}$ & 18.52 i & 24.40 abc & $4.64 \mathrm{e}$ & $6.12 \mathrm{~d}$ \\
\hline $\mathrm{M}_{1} \mathrm{G}_{0} \mathrm{~S}_{1}$ & 13.88 & 10.60 & $3.10 \mathrm{~d}$ & 23.93 & $86.50 \mathrm{j}$ & $23.40 \mathrm{~g}$ & 24.20 bcd & $3.96 \mathrm{i}$ & $5.02 \mathrm{~h}$ \\
\hline $\mathrm{M}_{1} \mathrm{G}_{0} \mathrm{~S}_{2}$ & 12.80 & 10.10 & $2.70 \mathrm{f}$ & 23.49 & $68.81 \mathrm{o}$ & $32.03 \mathrm{~b}$ & $23.77 \mathrm{~d}$ & $2.89 n$ & $3.58 \mathrm{l}$ \\
\hline $\mathrm{M}_{1} \mathrm{G}_{1} \mathrm{~S}_{0}$ & 15.00 & 11.20 & $3.80 \mathrm{a}$ & 24.50 & $103.1 \mathrm{c}$ & $15.83 \mathrm{j}$ & 24.43 abc & $4.96 \mathrm{c}$ & $6.49 \mathrm{~b}$ \\
\hline $\mathrm{M}_{1} \mathrm{G}_{1} \mathrm{~S}_{1}$ & 14.17 & 10.90 & $3.24 \mathrm{c}$ & 23.80 & $93.30 \mathrm{~h}$ & $21.63 \mathrm{~h}$ & 24.42 abc & $4.42 \mathrm{~g}$ & 5.98 ef \\
\hline $\mathrm{M}_{1} \mathrm{G}_{1} \mathrm{~S}_{2}$ & 13.19 & 10.36 & $2.83 \mathrm{e}$ & 23.60 & $76.33 \mathrm{~m}$ & $27.00 \mathrm{~d}$ & $24.01 \mathrm{~cd}$ & $3.32 \mathrm{l}$ & $4.43 \mathrm{j}$ \\
\hline $\mathrm{M}_{2} \mathrm{G}_{0} \mathrm{~S}_{0}$ & 15.12 & 11.30 & $3.82 \mathrm{a}$ & 24.30 & 105.7 b & $14.11 \mathrm{k}$ & 24.50 abc & $5.14 \mathrm{~b}$ & $6.60 \mathrm{~b}$ \\
\hline $\mathrm{M}_{2} \mathrm{G}_{0} \mathrm{~S}_{1}$ & 14.20 & 10.95 & $3.25 \mathrm{c}$ & 24.10 & $95.49 \mathrm{~g}$ & $21.07 \mathrm{~h}$ & 24.40 abc & $4.50 \mathrm{f}$ & $6.09 \mathrm{de}$ \\
\hline $\mathrm{M}_{2} \mathrm{G}_{0} \mathrm{~S}_{2}$ & 13.26 & 10.60 & $2.86 \mathrm{e}$ & 23.54 & $78.61 \mathrm{l}$ & $26.01 \mathrm{e}$ & $24.65 a b$ & $3.48 \mathrm{k}$ & $4.61 \mathrm{i}$ \\
\hline $\mathrm{M}_{2} \mathrm{G}_{1} \mathrm{~S}_{0}$ & 15.25 & 11.40 & $3.85 \mathrm{a}$ & 24.53 & 110.5 a & $12.00 \mathrm{l}$ & $24.60 \mathrm{ab}$ & $5.35 \mathrm{a}$ & $6.80 \mathrm{a}$ \\
\hline $\mathrm{M}_{2} \mathrm{G}_{1} \mathrm{~S}_{1}$ & 14.29 & 11.00 & $3.29 \mathrm{c}$ & 24.24 & 98.18 e & $18.50 \mathrm{i}$ & 24.45 abc & $4.67 \mathrm{e}$ & $6.37 \mathrm{c}$ \\
\hline $\mathrm{M}_{2} \mathrm{G}_{1} \mathrm{~S}_{2}$ & 13.70 & 10.80 & $2.90 \mathrm{e}$ & 23.75 & $86.72 \mathrm{j}$ & $24.04 \mathrm{~g}$ & $24.18 \mathrm{bcd}$ & $4.00 \mathrm{i}$ & $5.01 \mathrm{~h}$ \\
\hline F-test & NS & NS & $*$ & NS & $* *$ & $* *$ & $* *$ & $* *$ & $* *$ \\
\hline $\operatorname{LSD}_{(0 .}$ & - & - & 0.117 & - & 0.794 & 0.778 & 0.480 & 0.074 & 0.117 \\
\hline CV (\%) & 0.55 & 0.57 & 2.20 & 1.26 & 0.54 & 2.10 & 1.19 & 1.11 & 1.25 \\
\hline
\end{tabular}

In a column, figures with same letter or without letter do not differ significantly, whereas figures with dissimilar letters differ significantly (as per DMRT)

* = Significant at $5 \%$ level of probability

** $=$ Significant at $1 \%$ level of probability

NS = Not significant

$\mathrm{M}_{0}=0, \mathrm{M}_{1}=5$ ton green manure $/ \mathrm{ha}, \mathrm{M}_{2}=10$ ton green manure $/ \mathrm{ha}, \mathrm{G}_{0}=0 \mathrm{~g}$ gypsum $/ \mathrm{kg}$ soil, $\mathrm{G}_{1}=1 \mathrm{~g}$ gypsum $/ \mathrm{kg}$ soil, $\mathrm{S}_{0}=0$ $\mathrm{mM} \mathrm{NaCl}, \mathrm{S}_{1}=25 \mathrm{mM} \mathrm{NaCl}, \mathrm{S}_{2}=50 \mathrm{mM} \mathrm{NaCl}$

Table 7. Amelioration of salinity stress on grain yield and straw yield by interaction of gypsum application and green manuring

\begin{tabular}{c|c|c|c|c}
\hline $\begin{array}{c}\text { Interaction } \\
(\mathrm{M} x \mathrm{G} \text { x S })\end{array}$ & $\begin{array}{c}\text { Grain yield } \\
\left(\mathrm{t} \mathrm{ha}^{-1}\right)\end{array}$ & $\begin{array}{c}\text { \% increase or } \\
\text { decrease over } \\
\text { control }\end{array}$ & $\begin{array}{c}\text { Straw yield } \\
\left(\mathrm{t} \mathrm{ha}^{-1}\right)\end{array}$ & $\begin{array}{c}\text { \% increase or } \\
\text { decrease over } \\
\text { control }\end{array}$ \\
\hline $\mathrm{M}_{0} \mathrm{G}_{0} \mathrm{~S}_{0}$ & 4.49 & - & 5.92 & - \\
$\mathrm{M}_{0} \mathrm{G}_{1} \mathrm{~S}_{0}$ & 4.75 & 5.79 & 6.29 & 6.25 \\
$\mathrm{M}_{1} \mathrm{G}_{0} \mathrm{~S}_{0}$ & 4.64 & 3.34 & 6.12 & 3.37 \\
$\mathrm{M}_{1} \mathrm{G}_{1} \mathrm{~S}_{0}$ & 4.96 & 10.46 & 6.49 & 9.62 \\
$\mathrm{M}_{2} \mathrm{G}_{0} \mathrm{~S}_{0}$ & 5.14 & 14.47 & 6.6 & 11.48 \\
$\mathrm{M}_{2} \mathrm{G}_{1} \mathrm{~S}_{0}$ & 5.35 & 19.15 & 6.8 & 14.86 \\
$\mathrm{M}_{0} \mathrm{G}_{0} \mathrm{~S}_{1}$ & 3.86 & -14.03 & 5.06 & -14.52 \\
$\mathrm{M}_{0} \mathrm{G}_{1} \mathrm{~S}_{1}$ & 4.1 & -8.68 & 5.37 & -9.29 \\
$\mathrm{M}_{1} \mathrm{G}_{0} \mathrm{~S}_{1}$ & 3.96 & -11.80 & 5.02 & -15.20 \\
$\mathrm{M}_{1} \mathrm{G}_{1} \mathrm{~S}_{1}$ & 4.42 & -1.55 & 5.98 & 1.01 \\
$\mathrm{M}_{2} \mathrm{G}_{0} \mathrm{~S}_{1}$ & 4.5 & 0.22 & 6.09 & 2.87 \\
$\mathrm{M}_{2} \mathrm{G}_{1} \mathrm{~S}_{1}$ & 4.67 & 4.00 & 6.37 & -4.60 \\
$\mathrm{M}_{0} \mathrm{G}_{0} \mathrm{~S}_{2}$ & 2.61 & -41.87 & 3.307 & -33.27 \\
$\mathrm{M}_{0} \mathrm{G}_{1} \mathrm{~S}_{2}$ & 3.06 & -31.84 & 3.95 & -39.52 \\
$\mathrm{M}_{1} \mathrm{G}_{0} \mathrm{~S}_{2}$ & 2.89 & -35.63 & 3.58 & -25.16 \\
$\mathrm{M}_{1} \mathrm{G}_{1} \mathrm{~S}_{2}$ & 3.32 & -26.05 & 4.43 & -22.12 \\
$\mathrm{M}_{2} \mathrm{G}_{0} \mathrm{~S}_{2}$ & 3.48 & -22.49 & 4.61 &
\end{tabular}


Siam et al.

$\mathrm{M}_{2} \mathrm{G}_{1} \mathrm{~S}$ 4

$-10.91$

5.01 $-15.37$

$\mathrm{M}_{0}=0, \mathrm{M}_{1}=5$ ton green manure $/ \mathrm{ha}, \mathrm{M}_{2}=10$ ton green manure $/ \mathrm{ha}$

$\mathrm{G}_{0}=0 \mathrm{~g}$ gypsum $/ \mathrm{kg}$ soil, $\mathrm{G}_{1}=1 \mathrm{~g}$ gypsum $/ \mathrm{kg}$ soil

$\mathrm{S}_{0}=0 \mathrm{mM} \mathrm{NaCl}, \mathrm{S}_{1}=25 \mathrm{mM} \mathrm{NaCl}, \mathrm{S}_{2}=50 \mathrm{mM} \mathrm{NaCl}$

\section{Conclusion}

The results of the study reveal that combined application of green manure @ $10 \mathrm{t} \mathrm{ha}^{-1}$ and gypsum @ $1 \mathrm{~g}$ $\mathrm{kg}^{-1}$ soil ameliorated salinity stress in transplant Aman rice remarkably. The combination of application of green manure @ $10 \mathrm{t} \mathrm{ha}^{-1}$ and gypsum @ $1 \mathrm{~g} \mathrm{~kg}^{-1}$ soil increased the grain yield by 4\% in case of 25 $\mathrm{mM} \mathrm{NaCl}$ compared to control while grain yield decreased by $14.03 \%$ when no green manure and gypsum were applied. The combination also minimized the grain yield reduction from $41.87 \%$ to $10.91 \%$ in case of $50 \mathrm{mM} \mathrm{NaCl}$ salinity stress. To validate the result, however, similar experiment needs to be conducted under natural occurrence of salinity stress.

\section{References}

Flowers, T. J. and A. R. Yeo. 1981. Ion relations of plants under drought and salinity. Aust. J. Plant Physiol. 13: 75-91.

Gomez, K A. and, A. A. Gomez. 1984. Statistical Procedures for Agricultural Research. 2nd edn. John Wiley and sons, New York. p.680.

Hossain, M. A. 2002. Effect of salinity stress in some genotypes of rice. M. S Thesis Dept. Crop Bot., Bangladesh Agril. Univ., Mymensingh.

Hossain, M. M. 2006. Effect of salinity level on growth and yield of advanced mutant rice in Boro season. M. S. Thesis, Dept. Crop Bot., Bangladesh Agril. Univ. Mymensingh.

Islam, M. S., A. H. Molla and H. A. Quayum. 2004. Effect of different levels of salinity on grain filling in rice. Bull. Inst. Trop. Agric., Kyushu Univ. 24: 19-22.

Karim, Z., S. G. Hussain and, M. Ahmed. 1990. Salinity problems and crop intensification in the coastal regions of Bangladesh. Bangladesh Agricultural. Research Council (BARC). p.1.

Khattak, S. G., A.Izhar, M. J. Khattak and Naveedullah. 2007. Effect of various levels of gypsum application on the reclamation of salt affected soil. Pakistan. J. Sarhad Agric. 23 (3): 675-680.

Massoud, F. I. 1994. Salinity and alkalinity as soil degradation hazards, FAO/UNDP expert consolation on soil degradation, June 10-14, 1974, FAO, Rome. p.21.

Rashid, M. M. 2005. Effect of salinity at different growth stages of transplanted aman rice mutants. M. S Thesis, Dept. Crop Rot., Bangladesh Agril. Univ., Mymensingh.

Sen, A. K. 2002. Salinity tolerance studies of some transplanted aman rice cultivars. M. S Thesis. Dept. Crop Bot., Bangladesh Agril. Univ., Mymensingh.

Singh, Y. P., R. Singh and, D. K. Sharma. 2009. Combined effect of reduced dose of gypsum and salt tolerant varieties of rice (Oryza sativa). Indian J. Agron. 54(1): 24-28.

Singh,Y. P., R. Singh and N. Kumar. 2008. Response of rice (Oryza sativa) to gypsum rates in sodic soils. Indian J. Agric. Res. 78(4): 362-365.

Vakeesan, S. and T. Nishanthan. 2007. Effect of green manuring (Sesbania aculeata )and gypsum in submerged calcareous and noncalcareous soils. Biol.Fert. Soil. 4(3): 159-162. 
Salinity Stress on Transplant Aman Rice 
Siam et al. 